Revista de Investigación Educativa 27

julio-diciembre, 2018 | ISSN 1870-5308 | Xalapa, Veracruz

Instituto de Investigaciones en Educación | Universidad Veracruzana

\title{
Escritura colaborativa en entornos de formación virtual de una asignatura universitaria sobre Tecnología Educativa
}

\section{Collaborative writing in e-learning academic environments of a university course about Educational Technology}

\author{
Guadalupe Álvarez ${ }^{\mathrm{a}}$ \\ Lorena Bassa ${ }^{b}$ \\ Alejo González López Ledesmac
}

Recibido: 03 de abril de 2018

Aceptado: 17 de julio de 2018

En este artículo se aborda un tipo de práctica escrituraria que se ha vuelto frecuente en las comunidades académicas y profesionales: la escritura colaborativa mediada por tecnologías digitales. Nos centramos, específicamente, en el contexto universitario. El objetivo es caracterizar las prácticas de escritura colaborativa desarrolladas durante una cursada completamente virtual de la asignatura Tecnología Educativa de la Licenciatura en Educación de la Universidad Nacional de General Sarmiento, Buenos Aires, Argentina, a partir de una serie de categorías conceptuales propuestas en la bibliografía (estrategias para la escritura, fases y actividades, modos de control del documento, roles, modalidades de trabajo). Estas categorías resultan ajustadas y completadas durante el proceso analítico. En este sentido, nuestra principal conclusión es que, cuando se trata de contextos educativos, las dimensiones planteadas en la bibliografía para reflexionar sobre la escritura colaborativa en diferentes ámbitos se deberían articular con aspectos relativos al rol docente y las estrategias didácticas implementadas antes, durante y después del proceso escriturario.

Palabras clave: Elearning; educación universitaria; escritura; aprendizaje colaborativo.

\footnotetext{
a Doctora en Letras. Investigadora Adjunta, Consejo Nacional de Investigaciones Científicas y Técnicas, Argentina.凶 galvarez@ungs.edu.ar.

bEspecialista en Lectura, Escritura y Educación. Docente-Investigadora, Universidad Nacional de General Sarmiento, Argentina.凶 mbassa@campus.ungs.edu.ar.

'Especialista en Tecnología Educativa. Becario doctoral, Consejo Nacional de Investigaciones Científicas y Técnicas, Argentina.凶 alejoegll@gmail.com
} 
This paper examines a type of writing practice that has become frequent in the professional and academic community: collaborative writing with digital technology mediation. We specifically focus on the university context. The aim is to describe the collaborative writing practices of an online course of Educational Technology, a subject of the Licentiate in Education in the Universidad Nacional de General Sarmiento, Buenos Aires, Argentina, based on a series of conceptual categories selected from specialized literature (writing strategies, phases and activities, document control modes, roles and work methods). These categories are adjusted and completed during the analytical process. In this sense, our main conclusion is that, in educational contexts, the analyzed concepts developed in the literature to study collaborative writing in general should be articulated with dimensions related to the teachers' role and the didactic strategies involved before, during and after the writing process.

Key words: Elearning; high education; writing; collaborative learning.

\title{
Escritura colaborativa en entornos de formación virtual de una asignatura universitaria sobre Tecnología Educativa
}

\author{
Collaborative writing in e-learning academic environments \\ of a university course about Educational Technology
}

\section{Introducción}

$\mathrm{E}$ n el contexto de los estudios superiores, se ha vuelto cada vez más frecuente el uso de la tecnología digital como soporte de la enseñanza y el aprendizaje (Laurillard, 2010; Rama, 2007; Rodríguez \& Escofet, 2006; Torres \& Rama, 2010). De hecho, un estudio en Latinoamérica y el Caribe ha registrado que, ya en 2005, 201 instituciones universitarias llevan adelante el proceso de virtualización de la educación: 11\% orientado a la formación online pura; $89 \%$ restante, a modelos formativos que combinan elementos presenciales y no presenciales (Rama, 2007; Torres \& Rama, 2010). 
Frente a este panorama y asumiendo la importancia que la escritura adquiere en la universidad, proponemos indagar un tipo de práctica escrituraria que se ha vuelto frecuente en las comunidades académicas y profesionales: la escritura colaborativa mediada por tecnologías digitales. Nos preguntamos, en particular: ¿qué características asume el proceso de composición escrita cuando se lleva a cabo de manera grupal y con mediación tecnológica en el contexto universitario? A fin de ofrecer una respuesta inicial - aunque obviamente no acabada - a este interrogante, nuestro objetivo será caracterizar las prácticas de escritura colaborativa desarrolladas durante una cursada completamente virtual de la asignatura Tecnología Educativa de la Licenciatura en Educación de la Universidad Nacional de General Sarmiento (UNGS) (Buenos Aires, Argentina) a partir de una serie de categorías conceptuales creadas con estos fines por Posner y Baecker (1992), y Lowry, Curtis y Lowry (2004), a saber: estrategias para la escritura, fases y actividades, modos de control del documento, roles y modalidades de trabajo. Durante el proceso de análisis, estas categorías han sido ajustadas y completadas considerando dos categorías adicionales, teniendo en cuenta la reflexión llevada a cabo en relación al concepto de aprendizaje colaborativo (Dillenbourg, 1999, 2002): condiciones iniciales de las actividades de escritura grupal e intervenciones docentes durante el desarrollo de la actividad.

\section{Marco teórico}

Antes de comprender las conceptualizaciones desarrolladas sobre la escritura colaborativa, es fundamental reconocer el concepto de aprendizaje colaborativo.

\subsection{Del aprendizaje cooperativo al colaborativo}

Diversos investigadores han estudiado los rasgos del aprendizaje colaborativo (Dillenbourg, 1999, 2002; Stahl, Koschmann \& Stuthers, 2006), en algunos casos estableciendo diferencias con la cooperación (Kreijns, Kirschner \& Jochems, 2003).

Desde un punto de vista, se considera que no hay una evidente distinción entre la colaboración y la cooperación. Así, se postula que en ambos tipos de aprendizaje es fundamental que el estudiante desarrolle un tipo de aprendizaje activo, asumiendo responsabilidades sobre el propio proceso formativo, y que el docente desempeñe un 
rol facilitador. De esta manera, la enseñanza y el aprendizaje suelen representar experiencias compartidas (Kreijns et al., 2003).

Desde otra perspectiva, los investigadores proponen diferenciar el aprendizaje cooperativo del colaborativo y se interesan más por este último.

Siguiendo este enfoque, Stahl et al. (2006) consideran que mientras que en el trabajo cooperativo los alumnos llevan a cabo tareas individuales que combinan después en el producto final, en el trabajo colaborativo asumen juntos el desarrollo de una tarea común y, a fin de lograr este desarrollo, negocian de manera grupal los significados, lo que les permite construir el conocimiento colectivamente.

Asimismo, Dillenbourg $(1999,2002)$ diferencia la cooperación de la colaboración, teniendo en cuenta las características de la división del trabajo. En el trabajo cooperativo, se establece una división vertical de las tareas: los estudiantes elaboran subtareas de manera individual y luego los ensamblan en un resultado final. La distribución de las actividades resulta rígida. Por el contrario, en la colaboración, la división resulta horizontal: los alumnos desarrollan "juntos” la tarea. Así, se puede establecer cierto intercambio de roles al desarrollar la actividad.

Paralelamente a estas diferencias, Dillenbourg (1999) destaca que el aprendizaje colaborativo no representa ni un método ni, tampoco, un mecanismo. Se trata, más bien, de un tipo de contrato social que establecen profesores y estudiantes, por lo cual representa un contrato didáctico. De acuerdo con ello, el autor postula que la posibilidad de que se dé un tipo de trabajo colaborativo resulta mayor en los casos en que se establecen adecuadas condiciones iniciales (por ejemplo, el armado efectivo de grupos, el planteamiento anticipado de posibles problemas o el uso de programas informáticos apropiados) y también en las situaciones en que el docente se desempeña como facilitador orientando el desarrollo del trabajo. En función de ello, es posible contribuir con la colaboración de dos formas: mediante la organización inicial del proceso colaborativo o con la regulación posterior de su desarrollo (Dillenbourg, 2002).

El concepto de aprendizaje colaborativo ha sido recuperado para reflexionar sobre los fenómenos llevados a cabo en situaciones de escritura grupal, lo que ha llevado a postular la escritura colaborativa (EC) así como una serie de dimensiones básicas para su caracterización. 


\subsection{La escritura colaborativa mediada por tecnologías digitales}

La EC combina las tareas propias de la escritura individual (i.e., planificación, puesta en texto y revisión) con actividades (e.g. construcción de consenso) derivadas de la composición escrita grupal. Posner y Baeker (1992), primero, y Lowry et al. (2004), con posterioridad, han establecido categorías para analizar la práctica de escritura grupal: estrategias para la escritura, fases y actividades, modos de control del documento, roles, modalidades de trabajo.

Las estrategias para la escritura se establecen en función de la dinámica desarrollada en la producción textual: el grupo interactúa, pero un solo autor representa al equipo (escritura individual de intercambio grupal); diferentes integrantes escriben en distintos momentos (escritura individual secuencial); cada integrante escribe una sección del documento (escritura individual en paralelo); todos los miembros adoptan roles activos y no fijos (escritura interactiva); se combinan algunas de las instancias previamente mencionadas (modo mixto de escritura).

En cuanto a las fases y sus actividades, se suelen identificar tres fases en el proceso escriturario - preescritura, escritura propiamente dicha y posescritura- y diversas tareas asociadas a cada una: desde encuentros iniciales para organizar la dinámica del grupo hasta la entrega del documento final, pasando por la ideación, la planificación, el primer borrador, la revisión, la reescritura y la edición. Los modos de control se diferencian según el comportamiento que el grupo desarrolla al evaluar el escrito: se centraliza en un miembro; los miembros se turnan para la revisión; cada integrante revisa un "sub-documento", y todos los integrantes evalúan al mismo tiempo.

Los roles - escritor, editor, revisor, consultor, escriba - se establecen a partir del tipo de participación en la escritura. El editor puede modificar el contenido o el estilo, a diferencia del revisor, que sólo se ocupa de formalidades. El consultor no se integra al grupo, pero brinda comentarios que pueden optimizar el proceso. El escriba es quien sintetiza el escrito que se va elaborando a partir de la interacción del grupo.

Las modalidades de trabajo dependen de las variables de proximidad y sincronicidad, a saber: presenciales, asincrónicas, asincrónicas distribuidas y sincrónicas distribuidas. 
Escritura colaborativa en entornos de formación virtual de una asignatura universitaria sobre Tecnología Educativa

Guadalupe Álvarez, Lorena Bassa, Alejo González López Ledesma

\subsection{La escritura colaborativa mediada por tecnologías digitales en contextos académicos}

Los tipos de interactividad propia de los contextos digitales, así como el desarrollo de nuevas aplicaciones, ha favorecido la EC en contextos académicos. Esto ha llevado al desarrollo de investigación sobre el tema (Álvarez \& Bassa, 2013, 2016; Davioli, Monari \& Severinson, 2009; Guasch, Espasa \& Álvarez, 2010; Passig \& Schwartz, 2009), cuyos hallazgos resultan significativos para el presente trabajo.

En Collaborative Writing: online versus frontal, Passig y Schwartz (2007) asumen que la EC supone la elaboración de un texto acordada por todos los miembros de un grupo y, en función de esta conceptualización, proponen comparar las tareas colaborativas de escritura académica llevadas a cabo en forma presencial y en línea. Este estudio se desarrolla con diferentes grupos de dos o tres universitarios graduados con mucha experiencia en el uso de tecnologías digitales. Se utiliza Groove, un programa informático muy similar a Word, que permite la composición escrita en grupos, así como la comunicación de sus miembros a través de un chat. El programa también permite compartir las búsquedas realizadas en Internet y los documentos hallados. La evaluación de la composición escrita se llevó a cabo en función de diferentes criterios: 1) la calidad de la tarea; 2) la organización general del texto; 3) la pertinencia del lenguaje a la situación comunicativa; 4) el modo en que se construye la idea fundamental y la argumentación; 5) los aspectos gramaticales y la revisión textual, y 6) otros aspectos como las fuentes y la originalidad. El estudio muestra que se obtienen mejores resultados en cada uno de estos criterios cuando la escritura se realiza virtualmente. El criterio significativamente mejor es el relativo a la organización general. Parecería, entonces, que la tecnología favorece la coherencia del texto en tanto facilita las negociaciones y permite elaborar más borradores.

Asimismo, Davioli et al. (2009) analizan el uso de tres plataformas educativas diferentes - Moodle, Ping Pong y Blackboard-, en las cuales se desarrolla EC en situaciones que combinan clases presenciales y virtuales. Según los autores, los estudiantes refieren que la escritura es tanto más formal como más espontánea en las plataformas, lo cual podría deberse a que el medio en línea resulta más público, pero también menos directo, al no contar con la presencialidad. En cuanto a los comentarios, los estudiantes agregan que dedican más tiempo cuando la tarea es virtual. Otro hallazgo importante es que en el proceso de enseñanza-aprendizaje se percibe un rol docente diferente al habitual. 
Por otra parte, Guasch et al. (2010) estudian el efecto de la retroalimentación (feedback) en textos académicos producidos de manera colaborativa en entornos virtuales. Se consideran tres niveles en cuanto a la retroalimentación: dimensión funcional, dimensión semántica y dimensión estructural. Los resultados muestran que cuando las correcciones del docente son solamente informativas, los estudiantes no responden a la intervención, simplemente completan o corrigen el texto según las indicaciones. En los casos de las retroalimentaciones dadas por los docentes en las que hay sugerencias o propuestas de ampliación o revisión de oraciones, surgen cambios. Cuando los docentes plantean preguntas acerca del modo en que se pueden resolver cuestiones del texto, los estudiantes discuten sobre cómo mejorar sus ideas argumentativas. Además, elaboran respuestas y proponen discusiones para la reestructuración de ciertos episodios del texto, del mismo modo que pueden desarrollar argumentos a partir de abstracciones y contextualizaciones. Estos resultados corroboran e ilustran el valor del contexto grupal para la construcción conjunta de significados, y confirman la naturaleza epistémica de la retroalimentación que tiene lugar durante el proceso de escritura.

Por su parte, Álvarez y Bassa (2013) llevan a cabo una investigación cualitativa diseñada como estudio de caso descriptivo. Se centran en una materia ("Lengua”) del ingreso a la Universidad Nacional de Quilmes, particularmente en el blog utilizado en una de las comisiones de la asignatura. Dicha materia está orientada a la formación en lectura y escritura. En consonancia con ello, el blog funciona como un sitio en el que se publican los trabajos de los estudiantes y donde se proponen actividades de revisión. El análisis de la dinámica del blog, así como de las entrevistas a docentes y a estudiantes que participaron de su desarrollo, permite reconocer de qué manera el blog motiva a los alumnos y permite trabajar sobre dimensiones de la escritura que resultan difíciles de abordar en la presencialidad, como la edición del texto propio y la composición colaborativa de un escrito. Para que este tipo de actividades tenga lugar en el sitio, las actividades son diseñadas en función de un pasaje desde lo individual a lo cooperativo (participación sumativa de las intervenciones individuales) y de allí a lo colaborativo (participación horizontal de los diferentes miembros de los grupos).

Como continuidad de este estudio, Álvarez y Bassa (2016) proponen analizar actividades de escritura colaborativa llevadas a cabo en un taller de lectura y escritura del ingreso universitario. En esta asignatura los mismos estudiantes conformaron 16 grupos pequeños (de 2 a 4 integrantes) que podían optar por trabajar en papel o bien en documentos compartidos (DC, en adelante) de Google Drive (GD). El 75\% de los grupos eligió esta última aplicación. El análisis se lleva a cabo a partir de los conceptos de estra- 
tegias para la escritura, fases y actividades, modos de control del documento, roles, modalidades de trabajo (Lowry et al., 2004; Posner \& Baeker, 1992). En general, las autoras notan gran diversidad entre los grupos en el modo de organizar la producción del texto. De hecho, al interior de un mismo grupo se registran diversas estrategias para la escritura. También las autoras observan una correlación entre la coherencia y compleción de las producciones finales y la escritura individual de la totalidad, o una escritura individual a partir del intercambio grupal. En cuanto a las actividades de las diferentes fases del proceso escriturario, tras una planificación discutida en la clase presencial, varios grupos generan discusiones e intercambios en las actividades de ideación y la puesta en texto del primer borrador, lo que da lugar a mayor elaboración. Respecto de la revisión, se evidencian limitaciones en los casos en que el grupo asume la división horizontal, es decir, la escritura en paralelo de diferentes estudiantes. Del modo de control, las autoras aclaran que está determinado por la herramienta digital que habilita que todos puedan acceder al documento y revisarlo, aun cuando ello no ocurre en todos los grupos. En cuanto a los roles, es frecuente el escriba cuando los grupos desarrollan escritura individual tras el intercambio grupal. Por otra parte, un solo estudiante suele desempeñar el rol de revisor. En relación con las modalidades de trabajo, se recurre en general a intercambios sincrónicos utilizando diversos soportes tecnológicos (el DC, Skype, WhatsApp), en algunos casos herramientas que no han sido planificadas por el docente.

En el presente trabajo, continuaremos con la línea de investigación que se ha iniciado en estudios recién reseñados a fin de profundizar la caracterización de las prácticas de escritura colaborativa en la universidad.

\section{Consideraciones metodológicas}

\subsection{Contexto del estudio}

El estudio se llevó a cabo en el marco de Tecnología Educativa, una materia de la Licenciatura en Educación, carrera de cinco años del Instituto del Desarrollo Humano de la UNGS. Se trata de una asignatura semestral, con una carga horaria de 64 horas que se distribuyen semanalmente en bloques de 4 horas. En esta materia se propone un primer acercamiento o introducción al campo de convergencia entre las tecnologías, particularmente las nuevas tecnologías de la información y la comunicación (TIC), y la educación. 
La propuesta pedagógico-didáctica se articula en torno a dos estrategias. Por un lado, se toma distancia de la clase exclusivamente expositiva, centrada en el docente, para combinar las exposiciones con las actividades individuales y en grupos (pequeños y grandes). Se promueve, en este sentido, la transición desde instancias individuales a una aproximación de construcción colaborativa. Por otro lado, y en estrecha relación con lo anterior, se propicia un funcionamiento similar al de un taller: se apunta a instalar una dinámica que se mueve gradualmente desde una aproximación teórica hacia la intervención práctica de los propios estudiantes, pasando por la reflexión sobre casos reales de integración de TIC. En particular, se busca que los estudiantes experimenten con herramientas que favorezcan la producción y la inclusión de las tecnologías en la enseñanza.

En relación con las estrategias expuestas, es importante destacar que, a lo largo de la cursada, se proponen diferentes actividades que involucran la producción textual llevada a cabo por grupos pequeños.

El dictado de la asignatura es en general netamente presencial, con actividades de enseñanza y aprendizaje mediadas por tecnologías que se realizan en la clase o el hogar. Durante 2016, sin embargo, debido a una estadía de investigación en el exterior de la docente responsable, esta materia se dictó en modalidad completamente virtual.

Para el diseño y la implementación en línea, se recurrió a los espacios virtuales generados en la plataforma Moodle de la UNGs, los cuales se combinaron con otras aplicaciones (e.g. correo electrónico, documentos compartidos en GD, Prezi). Es importante destacar que se propuso una actividad, de realización individual o grupal, por semana, y en cada semana se presentaron todas las herramientas necesarias para el desarrollo de las tareas propuestas. Esto comprendió herramientas propias de Moodle (por ejemplo, un foro o una wiki) o de la Web 2.o.

\subsection{Diseño metodológico}

Durante la virtualización de Tecnología educativa, se recurrió al diseño de investigación de la Investigación-acción (IA) para contribuir a mejorar diversos aspectos del proceso de enseñanza-aprendizaje en esta asignatura universitaria, incluyendo la escritura.

Como explican Hernández, Fernández y Baptista (2006), la I A tiene como objetivo intervenir para mejorar una práctica situada a partir de aportar información para la revisión y la evaluación de una realidad que busca ser transformada. En este 
tipo de aproximación investigativa, el rol del investigador es sumamente proactivo: se trata en este caso de un investigador docente que está inmerso en la situación didáctica.

La IA se integra por diversas fases recursivas que comienzan con la detección del problema de la investigación y su clarificación en un diagnóstico, para luego formular un plan o programa con el que se va a intervenir la problemática detectada. Luego, este plan es implementado y se evalúan sus resultados. La naturaleza cíclica del diseño apunta a que estos resultados retroalimenten un nuevo diagnóstico para dar lugar a una nueva espiral de reflexión y acción.

En el presente artículo, nos centramos en un ciclo de la investigación y analizamos una dimensión específica del diseño y la implementación de la experiencia educativa: la escritura grupal y colaborativa. En este sentido, seleccionamos dos actividades de producción textual en pequeños grupos: la primera actividad, a la que nos referiremos como EC1, fue propuesta al inicio de la cursada; la segunda, que identificamos como EC2, fue planteada con posterioridad, luego de que los estudiantes hubieran realizado otras producciones individuales y grupales.

Para cuidar la identidad de los participantes, los nombres han sido modificados.

El camino de indagación metodológico comprendió dos etapas básicas:

\section{1) Recolección de datos}

Teniendo en cuenta que los datos adquieren mayor riqueza y profundidad si provienen de diversas fuentes y son obtenidos con variados métodos de recolección (Hernández et al., 2006), hemos considerado diferentes métodos para su relevamiento: a) registro documental y caracterización de las consignas diseñadas por los docentes; b) registro del desempeño de los estudiantes y de los docentes durante las actividades, y c) entrevista a los estudiantes sobre el desarrollo de las actividades. En relación con el registro del desempeño y las entrevistas, se tuvieron en cuenta los conceptos relativos a la escritura colaborativa.

\section{2) Análisis y delimitación teórica}

Una vez realizado el relevamiento recién mencionado, se ha llevado a cabo el análisis manualmente desde una perspectiva cualitativa. En esta instancia, dos investigadoras formadas examinaron cuidadosamente los datos provenientes de las diferentes fuen- 
tes a fin de observar el desempeño de los diversos grupos en las tareas de escritura colectiva. Este análisis comprendió dos momentos.

En un primer momento, se han destacado las similitudes y minimizado las diferencias del trabajo de los distintos grupos con el objeto de verificar las categorías conceptuales de las cuales se ha partido, fundamentalmente las siguientes: estrategias para la escritura, fases y actividades, modos de control del documento, roles, modalidades de trabajo (Lowry et al., 2004; Posner \& Baeker, 1992).

En la Tabla 1 se presentan las categorías y los indicadores del primer momento del análisis.

Tabla 1. Categorías e indicadores del primer momento de análisis

\begin{tabular}{|c|c|}
\hline Categorías & Indicadores \\
\hline \multirow{2}{*}{$\begin{array}{l}\text { Estrategia de } \\
\text { escritura* }\end{array}$} & $\begin{array}{l}\text { Tipo de agrupamiento en torno a la responsabilidad de la escritura (individual, } \\
\text { grupal). }\end{array}$ \\
\hline & $\begin{array}{l}\text { Dinámica de trabajo para la escritura (secuencial, interactiva, en paralelo, } \\
\text { modo mixto). }\end{array}$ \\
\hline \multirow{2}{*}{$\begin{array}{l}\text { Fases y } \\
\text { actividades }\end{array}$} & $\begin{array}{l}\text { Fase del proceso escriturario (preescritura, escritura propiamente dicha y } \\
\text { posescritura). }\end{array}$ \\
\hline & Tarea involucrada (entre ellas, encuentros presenciales o electrónicos al inicio). \\
\hline \multirow{3}{*}{$\begin{array}{l}\text { Modos de control } \\
\text { del documento }\end{array}$} & $\begin{array}{l}\text { Tipo de agrupamiento en torno a la responsabilidad del control (individual, } \\
\text { grupal). }\end{array}$ \\
\hline & Sujetos involucrados (docentes, docentes y alumnos, alumnos). \\
\hline & $\begin{array}{l}\text { Dinámica de trabajo en el control (centralizada, secuencial, interactiva y } \\
\text { simultánea, por secciones). }\end{array}$ \\
\hline \multirow{2}{*}{ Roles } & Sujeto en el rol (docentes o estudiantes). \\
\hline & Tipo de rol (escritor, editor, revisor, consultor y escriba). \\
\hline \multirow{2}{*}{$\begin{array}{l}\text { Modalidad de } \\
\text { trabajo }\end{array}$} & Canal (presencial, no presencial). \\
\hline & Modalidad (asincrónicas, asincrónicas distribuidas o sincrónicas distribuidas). \\
\hline
\end{tabular}

* En relación con esta dimensión, entonces, se ha reconocido si había interacción grupal, pero con un solo estudiante como responsable (escritura individual de intercambio grupal); si distintos estudiantes escribían en diferentes momentos (escritura individual secuencial); si cada integrante redactaba una sección del escrito (escritura individual en paralelo); si todos los integrantes adoptaban roles activos e intercambiables (escritura interactiva); o si se combinaban diferentes instancias de las mencionadas (modo mixto de escritura).

Fuente: Elaboración propia sobre la base de Lowry et al. (2004). 
En un segundo momento, se ha realizado el movimiento inverso resaltando las diferencias a fin de determinar las propiedades de dichas categorías. De esta manera, se ha ajustado y completado la categorización inicial. Se han añadido, en este sentido, dos categorías: "condiciones iniciales de las actividades de escritura grupal" e "intervenciones docentes durante el desarrollo de la actividad".

En la Tabla 2 se presentan las categorías y los indicadores añadidos en un segundo momento del análisis.

\section{Tabla 2. Categorías e indicadores añadidos en el segundo momento del análisis}

\begin{tabular}{ll}
\hline \multicolumn{1}{c}{ Categorías } & \multicolumn{1}{c}{ Indicadores } \\
\hline $\begin{array}{l}\text { Condicionales iniciales de las } \\
\text { actividades de escritura grupal }\end{array}$ & $\begin{array}{l}\text { Formas de decisión respecto de la conformación de los } \\
\text { grupos de trabajo. }\end{array}$ \\
\cline { 2 - 2 } $\begin{array}{l}\text { Intervenciones docentes durante el } \\
\text { desarrollo de la actividad }\end{array}$ & Fase de participación durante el proceso escriturario. \\
\cline { 2 - 2 } & Forma de participación. \\
\hline
\end{tabular}

Fuente: Elaboración propia.

Durante este proceso analítico, se ha considerado bibliografía específica para sistematizar las categorías y sus propiedades. Así, además de la bibliografía relativa a la escritura colaborativa, se ha recurrido a material bibliográfico desarrollado en torno al aprendizaje colaborativo (Dillenbourg, 1999, 2002).

De esta manera, por el camino del método comparativo, se ha generado teoría en función de las categorías ajustadas, lo que resulta de gran interés, puesto que permite comprender la realidad privilegiando la "densidad conceptual" antes que la "descripción densa" (Vasilachis, 2006).

\section{Resultados}

\subsection{Condiciones iniciales de las actividades de escritura grupal}

Es importante conocer las condiciones iniciales de las actividades, tanto las relativas a las consignas como a la conformación de los grupos. 
En la EC1, los estudiantes, a partir de conceptos de la bibliografía sobre políticas educativas - en particular, Buckingham (2008) y Tedesco (2008) - tuvieron que analizar tres políticas sobre inclusión de TIC en educación. Esta tarea consistió en la confección grupal de un cuadro en el cual se proponía comparar esas políticas en función de sus objetivos sociales, sus objetivos pedagógicos y el carácter tecnotópico. Para confeccionar este cuadro, los docentes habilitaron un documento compartido en GD y, para interactuar en torno a la tarea, un foro en el espacio Moodle de la asignatura.

En la EC2, los estudiantes, también en pequeños grupos, debían redactar un texto de aproximadamente 20 líneas a fin de exponer el análisis que realizaban de tres experiencias educativas con inclusión de tecnologías que los docentes de la asignatura les habían propuesto. El análisis tenía que contemplar el marco conceptual de bibliografía específica — González López \& Álvarez (2014), Maggio (2012)—, centrada en la inclusión de tecnologías digitales en el campo educativo. Para esta tarea, los docentes habilitaron tres herramientas en el espacio Moodle de la asignatura: wiki, foro y chat.

Las consignas de ambas actividades exponían, inicialmente, los casos por analizar; a continuación, el material bibliográfico por tener en cuenta, y, finalmente, especificaciones sobre el modo de desarrollar las tareas de redacción. En este sentido, es importante destacar que la EC1 no incluyó detalles sobre la dinámica grupal, a diferencia de la EC2, en la cual se dieron precisiones sobre esta dinámica. En particular, se ofrecieron alternativas para llevar a cabo la tarea, ya sea discutiendo en forma grupal el análisis y asignando algunas personas para la escritura y otras para la revisión, o discutiendo y escribiendo entre todos el análisis.

En relación específicamente con la conformación de los grupos de trabajo, es importante aclarar que, al comienzo de la cursada, se solicitó a los estudiantes que se agruparan por afinidad. Se conformaron de esta manera cuatro grupos con dos, tres o cuatro miembros. Nos referiremos a ellos con $1 \mathrm{G}_{1}, 1 \mathrm{G}_{2}, \mathrm{G}_{3}$ y ${ }_{1} \mathrm{G}_{4}$. No obstante, tras la primera actividad, los docentes recomendaron unificar dos de los grupos (el de tres con el de dos miembros). Así, quedaron conformados tres grupos (dos con cuatro integrantes y uno con cinco) que se mantuvieron durante toda la cursada, inclusive para la elaboración del trabajo final. Nos referiremos a ellos con $2 \mathrm{G}_{1}, 2 \mathrm{G} 2$ y $2 \mathrm{G}_{3}$.

A continuación, analizaremos las formas en que los grupos han desarrollado las actividades teniendo en cuenta los conceptos ya enunciados. 


\subsection{Estrategias para la escritura}

Respecto de las estrategias escriturarias y en función de lo propuesto por Posner y Baeker (1992) y Lowry et al. (2004), observamos que, en la EC1, todos los grupos organizaron la tarea en función de la estrategia de la escritura en paralelo (con división horizontal del trabajo). De esta manera, distribuyeron una política por alumno (cada dos en algunos pocos casos), y estas personas se encargaron del análisis de la política asignada y de su posterior redacción.

En la EC2, en cambio, todos los estudiantes intervinieron en las diferentes fases y tareas intercambiando roles de manera activa. Así, inicialmente negociaron en el foro la elección de la experiencia educativa para el análisis. En este sentido, se puede observar el Ejemplo 1.

Ejemplo 1 (correspondiente al $2 \mathrm{G}_{3}$ )

Re: Actividad 4.1: Foro del Grupo 3

de Valeria Sanchez - Tuesday, 4 de October de 2016, 23:56

Hola nuevamente!

No sé cómo quieren que nos organicemos con el análisis y luego la redacción del texto final de 20 renglones.

Por ahí, Pato te gustaría realizar la edición final?

Espero pronta respuesta.

vale

Según los propios estudiantes han declarado, en ocasiones la selección de experiencia se realizaba utilizando modalidades alternativas a las herramientas y aplicaciones propuestas por los docentes involucrando encuentros presenciales o contactos vía WhatsApp. Luego, lo acordado sobre la selección era reproducido en los foros. Una vez seleccionada la experiencia, iniciaban la negociación tanto de la estrategia de escritura y de revisión, como del contenido en sí. De este modo, se puede conceptualizar esta estrategia de escritura como de modo mixto y por momentos interactiva, ya que todos los integrantes se involucraron en el proceso.

\subsection{Fases y actividades}

En cuanto a las fases y actividades, en la EC1, como hemos anticipado, las tareas de planificación y escritura en sí estuvieron distribuidas horizontalmente, por lo que uno o dos alumnos se encargaron de una política. 
En la EC2, por el contrario, los alumnos compartieron ideas en el foro y negociaron luego allí el contenido que, tras un acuerdo, fue reproducido en la wiki. Es importante señalar que en ocasiones los estudiantes no coincidían inicialmente sobre el contenido del análisis, lo que generaba debates y más negociaciones.

En relación con la negociación que realizaron los estudiantes sobre el contenido, es importante también señalar que, en tanto la virtualidad exige necesariamente que se textualice cada mensaje de la manera más clara posible para que sea comprensible para todos, varios de los mensajes que fueron textualizados se integraron posteriormente a las versiones del documento de análisis. Así, a partir del intercambio entre pares se explicitó la ideación a partir de la cual se generaron los argumentos que se desplegaron en el trabajo final. La escritura como modo de interacción en la virtualidad aporta, entonces, su función epistémica ${ }^{1}$ a la construcción y objetivación de conocimiento, en esta ocasión, en torno al análisis de un caso a partir de reflexiones teóricas. Podemos relacionar esto con la función epistémica de la retroalimentación docente que proponen Guasch et al. (2010), que en esta experiencia está referida a la función epistémica que adquiere la retroalimentación escrita entre pares en la EC mediada virtualmente.

En relación con lo recién expuesto, es importante destacar que, tal como se señalara en los antecedentes analizados en el marco teórico, la distribución horizontal que hemos observado en la EC2 contrasta con la estrategia de planificación grupal que apunta a promover una discusión participativa previa a la escritura (por ejemplo, en la experiencia analizada en Álvarez \& Bassa, 2016). Según señalan estas autoras, el intercambio sobre la selección de información y la estructuración del texto lleva a una mayor elaboración de las ideas desplegadas en el trabajo final. Correlativamente, en EC1 la distribución horizontal de las fases de planificación y escritura resulta en producciones menos coherentes a nivel global que en las de la EC2.

En el próximo apartado incluimos detalles sobre el modo en que se ha llevado la revisión.

1. La función epistémica de la escritura hace referencia a la posibilidad que ofrece esta actividad para el trabajo intelectual y el desarrollo de operaciones cognitivas de orden superior (Bereiter \& Scardamalia, 1987; Flower, 1979; Flower \& Hayes, 1996). Desde este punto de vista, la escritura permitiría generar conocimiento y no solamente reproducirlo. 


\subsection{Modos de control del documento}

En ambas experiencias, las posibilidades relativas al modo de control del documento están condicionadas por las aplicaciones digitales seleccionadas (documentos compartidos en GD y wiki de Moodle), en tanto todos los participantes pueden acceder y eventualmente modificar lo escrito. De todos modos, se reconocen importantes diferencias en cuanto a la modalidad de la revisión que se pone en juego en ambas actividades.

En la EC1, en las tareas de valoración de lo analizado respecto de cada política, intervinieron al inicio los docentes con preguntas y/o sugerencias, valoraciones que invitaban a los alumnos a volver sobre lo realizado y ajustarlo. En este sentido, se puede leer el Ejemplo 2:

Ejemplo 2 (correspondiente al $1 \mathrm{G}_{1}$ )

María Lobos

29 Aug 2016

Habría que aclarar en qué sentido lo descripto muestra el carácter tecnotópico del proyecto. Para ello, pueden tener en cuenta la bibliografía leída y las preguntas propuestas en el documento base.

A partir de los comentarios docentes, cada alumno en general interactuaba con los docentes y ajustaba la política que inicialmente se le había asignado, tal como muestra el Ejemplo 3:

Ejemplo 3 (correspondiente al $1 \mathrm{G}_{2}$ )

Mario Dumas 29 Aug 2016

Resolve

Una buena pregunta para orientar el análisis del PCI a partir de los conceptos aportados por la bibliografía sería la siguiente: ¿Qué relación existe entre las dimensiones planteadas por Tedesco y los ejes del PCI?

\section{Flavia Álvarez}

31 Aug 2016

Me parece que podríamos pensarlo según lo que Tedesco presenta, las dimensiones social y política de las TIC como potenciadora de la posibilidad de mayor autonomía en el proceso de aprendizaje y construcción del conocimiento. 
Escritura colaborativa en entornos de formación virtual de una asignatura universitaria sobre Tecnología Educativa

Guadalupe Álvarez, Lorena Bassa, Alejo González López Ledesma

Mario Dumas

31 Aug 2016

Muy bien planteado, Flavia. En el PCI, entonces, habría una dimensión claramente social, vinculada a lo que él llama la brecha digital, y otra pedagógica, vinculada a la enseñanza, el aprendizaje y la construcción del conocimiento.

Muy pocos alumnos participan con comentarios en secciones de compañeros y, si lo hacen, es porque esto ha sido sugerido por los docentes.

A diferencia de la EC1, en la EC2 distintos estudiantes del grupo revisaron lo redactado en diferentes secciones, escritas por ellos o sus pares. Esto se evidencia, por ejemplo, en el siguiente intercambio:

Ejemplo 4 (correspondiente al $2 \mathrm{G}_{1}$ )

Re: Actividad 4.1: Foro del Grupo 1

de Lorena Gomez - Sunday, 2 de October de 2016, 19:56

Chicas: creo que destacamos los aspectos más relevantes de la propuesta b. Solo faltaría una voluntaria para organizar la información y en caso de ser necesario agregar algún dato pertinente. Pero recuerden que la extensión del trabajo es de 20 renglones. Saludos

Re: Actividad 4.1: Foro del Grupo 1

de Valeria Luna - Sunday, 2 de October de 2016, 23:30

Hola Chicas buenísimo, agrego y saco lo necesario.

Como se puede observar, en línea con lo analizado por Álvarez y Bassa (2013), la revisión no es una fase de la escritura que los estudiantes puedan abordar sin alguna guía o acompañamiento. Entendemos que, en esta experiencia, en Ec1 este andamiaje está dado por la intervención docente. A su vez, en EC2 se evidencia la apropiación del control de esta fase dado que los estudiantes ya han incorporado la relevancia de dicha fase y pueden realizarla con la guía del intercambio entre pares.

\subsection{Roles adoptados por integrantes de los grupos}

En la EC1, como se deduce de lo señalado en el resto de las categorías, los estudiantes adoptaron los roles de escritor y revisor en relación con la política que se le había asignado. Los docentes funcionaron como un tipo de consultores expertos sugiriendo no solamente modificaciones relativas a los análisis realizados, sino también modalidades de participación de los estudiantes. Y es sólo a partir de este tipo de 
sugerencias que algunos estudiantes revisaron lo escrito por otros miembros del grupo.

A diferencia de la EC1, en la EC2 los estudiantes adoptaron diferentes roles en relación con las diferentes fases y las diferentes secciones del escrito. Incluso, los comentarios sobre la revisión fueron realizados tanto por los docentes como por los propios alumnos.

En este sentido, como ya mencionamos, parece fundamental que, al menos en las primeras actividades propuestas, el docente asuma el rol de consultor externo para luego gradualmente fomentar que los integrantes del grupo también puedan ubicarse en un rol análogo.

\subsection{Modalidades de trabajo de los grupos}

Respecto de las modalidades de trabajo de los grupos, destacamos algunos hallazgos que nos resultan relevantes. En la EC1 los alumnos no usaron el foro habilitado por los docentes para estos fines, probablemente porque lo único que negociaron fue la asignación de política por alumno, lo que los estudiantes, según han declarado, resolvieron en encuentros presenciales o por intercambios de WhatsApp a los cuales los docentes no tuvieron acceso.

En la EC2, en cambio, los alumnos utilizaron el foro y el chat habilitados por los docentes para el intercambio, además de, como han expresado, las conversaciones presenciales o vía WhatsApp que han entablado sin que los docentes pudieran acceder a ello.

\subsection{Intervenciones docentes durante el desarrollo de la escritura grupal}

En este parágrafo daremos cuenta de una categoría que entendemos necesaria y complementaria a las anteriores (las desarrolladas de 3.2 a 3.6), más estrictamente relacionadas a distintas taxonomías propuestas para analizar la EC (Lowryet al., 2004; Posner \& Baeker, 1992). Así, aunque las intervenciones docentes han sido analizadas en función de los parámetros ya expuestos, consideramos importante delimitarlas como una categoría independiente por su decisiva incidencia en las demás.

Como señalan Davioli et al. (2009), la modalidad virtual implica un rol docente que se configura de un modo diferente a la presencialidad. En particular, resulta significativo considerar el principio de ajuste de la ayuda de Onrubia (2005) para dar 
cuenta de las estrategias del docente virtual que buscan promover la capacidad de autonomía y de autorregulación del estudiante. Esta autonomía supone en la EC la capacidad de interactuar y negociar activamente en distintos roles, fases y actividades del proceso escriturario.

En este sentido es que, según observamos, en la EC1 la intervención docente se realizó, por un lado, en la configuración de distribución horizontal de la consigna. Y si bien esto hubiera podido desalentar la interacción entre pares, ha permitido una primera aproximación ordenada a una producción colectiva, en la que se garantizó que cada integrante de un equipo participara. Por otro la, los docentes intervinieron con sus comentarios de reflexión sobre los avances y, según entendemos, prepararon a los estudiantes para una posterior actividad que suponía mayor reflexión e intercambio entre pares.

Esto efectivamente se concretó en EC2, junto con una mayor amplitud de roles y fases en las que participó cada estudiante.

Dado que no podemos suponer que los estudiantes hayan tenido experiencias previas de EC mediadas por tecnologías en el ámbito académico, es que valoramos la gradualidad progresiva de EC1 a EC2. Sin embargo, restaría indagar sobre formas de lograr que los estudiantes realicen una reflexión explícita sobre su progresiva conformación como escritores colaborativos plenos.

\section{Conclusiones}

En el presente artículo caracterizamos prácticas de escritura grupal en contextos universitarios, particularmente dos actividades identificadas como EC1 y EC2, que fueron desarrolladas durante una cursada virtual de la materia Tecnología Educativa de la UNGS: la primera al comienzo de la cursada y la segunda luego de ya realizados diversos trabajos individuales y grupales. El análisis se realizó en función de una serie de categorías conceptuales iniciales propuestas en la bibliografía (Lowry et al. 2004; Posner \& Baeker, 1992) para reflexionar sobre el proceso de escritura colaborativo, particularmente las estrategias para la escritura, las fases y actividades, los modos de control del documento, los roles y las modalidades de trabajo. Tras un proceso de análisis y delimitación teórica, estas categorías fueron ajustadas y completadas en función de conceptualizaciones provenientes de los estudios sobre el aprendizaje colaborativo (Dillenbourg, 1999, 2002). En este sentido, se consideraron dos categorías 
adicionales: las condiciones iniciales de la actividad de escritura grupal y las intervenciones docentes durante la actividad.

El análisis realizado pone en evidencia diferencias significativas entre la EC1 y la EC2. En la primera actividad, la estrategia de escritura, caracterizada por una distribución horizontal, determina que, una vez que se ha distribuido una sección del escrito por integrante, cada uno se ocupe de la escritura y la revisión del escrito. La distribución de las tareas resulta, entonces, rígida y sólo se modifica levemente ante las sugerencias de los docentes, que se desempeñan como consultores expertos. En la EC2, en cambio, todos los integrantes intervienen tanto en la negociación sobre la distribución de las tareas como en las discusiones relativas al contenido, la textualización y la revisión del análisis.

Resulta significativo, en este sentido, retomar las condiciones iniciales propuestas en la consigna diseñada por el docente. En el primer caso, no se establece ningún tipo de recomendación sobre el modo de realizar la actividad, mientras que en el segundo se plantean al menos dos alternativas que implican una distribución más equitativa de las tareas y los roles. Este hallazgo parecería indicar que, como sostiene Dillenbourg (1999), la posibilidad de que se den interacciones más colaborativas se puede incrementar a partir de buenas condiciones planteadas inicialmente por el docente en relación, entre otros criterios, con el armado de los grupos, el uso de aplicaciones, la posibilidad de resolución de problemas.

Paralelamente, se puede dar lugar a interacciones más colaborativas de manera retroactiva, a partir de intervenciones en las actividades (Dillenbourg, 2002). En este sentido, como muestra claramente la EC1, el docente puede intervenir en caso de distribuciones horizontales a fin de que se genere mayor interacción entre los integrantes de los grupos.

Por otra parte, tomando como eje de análisis las intervenciones docentes, se puede evidenciar una secuenciación entre $\mathrm{EC} 1$ y EC2 que supone un progresivo ajuste de la ayuda (Onrubia, 2005) del docente para fomentar mayor participación colaborativa de los estudiantes en las diversas fases, actividades y roles del proceso. A pesar de esto, faltaría proponer una reflexión explícita sobre el proceso de Ec, lo cual creemos que potenciaría su transferencia a otras situaciones.

Los resultados del análisis permiten deducir que, cuando se trata de analizar actividades de escritura colaborativa en contextos educativos virtuales, las dimensiones planteadas en la bibliografía para reflexionar sobre estas prácticas escriturarias en general deberían articularse con un aspecto relativo al rol docente y las estrategias 
didácticas antes, durante y después del proceso escriturario. En este sentido, es que entendemos que el docente en la consigna debe pautar ciertos aspectos que habiliten la interacción no sólo en torno al contenido y su construcción (es decir, generar discusión metacognitiva), sino también acerca de cómo se organiza la tarea grupal. Así, como en $\mathrm{EC}_{2}$, la tarea precisa de negociación respecto de la dinámica grupal y prevé espacios de discusión que garantizan la etapa de preescritura.

Vinculado a esta etapa, destacamos el hecho de que los intercambios en torno a la ideación y la discusión inicial se realicen a través de la escritura digital —enmarcada en un contexto universitario- - Esto permite subrayar (y potenciar) la función objetivadora y epistémica de la escritura, ya que el conocimiento que se produce de manera más dialogal en el intercambio, luego se consolidará de modo más consensuado en el producto final, como efectivamente ha sucedido en EC2. Esta explicitación del proceso de elaboración de un análisis terminado (propio de la escritura colaborativa) queda entonces registrado en los espacios virtuales y fortalece aún más a la escritura en sí como tecnología cognitiva.

Por otra parte, hemos observado que, más allá de las diferencias que presentan la EC1 y la EC2, ambas ponen en evidencia un hallazgo ya reconocido en estudios previos (Álvarez \& Bassa, 2016): los grupos construyen entornos diversos de comunicación y producción que, en general, amplían lo pautado por los docentes. Y, de hecho, como declaran los estudiantes en entrevistas sobre lo realizado, en ocasiones ellos prefieren establecer interacciones en espacios "no oficiales", lo que lleva a que no sean utilizadas las herramientas habilitadas para las actividades por los profesores. Esta observación lleva a dejar planteados algunos desafíos de la investigación sobre enseñanza de la lectura y la escritura mediadas por tecnologías digitales. En particular, interesa poner de relieve la importancia de considerar diferentes métodos de recolección de datos más allá de la observación y el registro de la actividad de los estudiantes que esté mediada por aplicaciones propuestas por docentes.

En definitiva, la categorización aplicada parecería resultar exhaustiva para el corpus analizado en cuanto los indicadores definidos a priori han abarcado las particularidades empíricas concretas de la escritura grupal en el contexto universitario analizado. De esta manera, a partir del análisis realizado, nos hemos aproximado a la comprensión de la escritura colaborativa en el marco de la formación virtual universitaria. De todos modos, consideramos que se requiere ampliar el análisis a fin de contar con datos suficientes como para sugerir estrategias didácticas que permitan optimizar las prácticas de escritura colaborativa mediadas por tecnologías en la universidad. 


\section{Lista de referencias}

Álvarez, G., \& Bassa, L. (2013). TIC y aprendizaje colaborativo: el caso de un blog de aula para mejorar las habilidades de escritura de los estudiantes preuniversitarios. RUSC. Revista de Universidad y Sociedad del Conocimiento, 10(2), 5-19. Recuperado de http://dx.doi.org/10.7238/rusc.v10i2.1740

Álvarez, G., \& Bassa, L. (2016). Estrategias didácticas para promover la escritura colaborativa mediada por tecnologías: hacia el desarrollo de dinámicas expertas en los grupos de trabajo. Exlibris, 5, 242-247. Recuperado a partir de http:// revistas.filo.uba.ar/index.php/exlibris/article/view/3022/968

Bereiter, C., \& Scardamalia, M. (1987). The Psychology of Written Composition. Nueva Jersey: Erlbaum.

Buckingham, D. (2008). Más allá de la tecnología: aprendizaje infantil en la era de la cultura digital (Trad. E. Odiozola). Buenos Aires: Manantial S.R.L.

Davioli, P., Monari, M., \& Severinson, K. (2009). Peer Activities on Web-Learning Platforms. Impact on Collaborative Writing and Usability Issues. Education and Information Technologies, 14(3), 229-254. doi:10.1007/s10639-008$9080-\mathrm{x}$

Dillenbourg, P. (Ed.). (1999). Collaborative-learning: Cognitive and Computational Approaches. Oxford: Elsevier.

Dillenbourg, P. (2002). Over-scripting CSCL: The risks of blending collaborative learning with instructional Design. En P. A. Kirschner (Ed.), Three Worlds of CSCL: Can we Support CSCL? (pp. 61-92). Heerlen: Open Universiteit.

Flower, L. (1979). Writer-based prose: A cognitive basis for problems in writing. College English, 41(1), 19-37.

Flower, L., \& Hayes, J. (1996). Teoría de la redacción como proceso cognitivo. Textos en contexto, 73-110.

González López, A., \& Álvarez, G. (2014) Tipología de propuestas de abordaje de contenidos de Lengua y Literatura con inclusión de TIC. Apertura, Revista de Innovación Educativa, 6(2). Recuperado de http://www.udgvirtual.udg.mx/ apertura/index.php/apertura/article/view/531/367

Guasch, T., Espasa, A., \& Álvarez, I. (2010). Formative e-feedback in collaborative writing assignments: the effect of the process and time. eLC. Research Paper Series, 1, 49-59. Recuperado de http://elcrps.uoc.edu/elcrps/index.php/elcrps/article/view/issue1-guasch-espasa-alvarez.html 
Hernández, R., Fernández, C., \& Baptista, P. (2006). Metodología de la Investigación ( $4^{\text {a }}$ ed.). México: Mc Graw Hill.

Kreijns, K., Kirschner, P., \& Jochems, W. (2003). Identifying the pitfalls for social interaction in computer-supported collaborative learning environments: a review of the research. Computers in Human Behavior, 19, 335-353.

Laurillard, D. (2010). Effective use of technology in teaching and learning in he. International Encyclopedia of Education, 4, 419-426.

Lowry, P., Curtis, A., \& Lowry, M. (2004). Building a Taxonomy and Nomenclature of Collaborative Writing to Improve Interdisciplinary Research and Practice. International Journal of Business Communication, 41-66.

Maggio, M. (2012). Enriquecer la enseñanza. Los ambientes con alta disposición tecnológica como alta oportunidad. Buenos Aires: Paidós.

Onurbia, J. (febrero, 2005). Aprender y enseñar en entornos virtuales: actividad conjunta, ayuda pedagógica y construcción del conocimiento. RED. Revista de Educación a Distancia 2 (número monográfico). Recuperado de http://www. um.es/ead/red/M2/

Passig, D., \& Schwartz, G. (2007). Collaborative Writing: Online versus Frontal. International Journal on e-Learning, 6(3), 395-412.

Posner, I. R., \& Baecker, R. M. (1992). How people write together. En IEEE Computer Society (Ed.), Proceedings of the 25th Hawaii International Conference on System Sciences (Vol. IV, pp. 127-138). Washington: Editor.

Rama, C. (2007). La despresencialización de la educación superior en América Latina. ¿Tema de calidad, de cobertura, de internacionalización o de financiamiento? Apertura, 7(06), 32-49.

Rodríguez, J. L., \& Escofet, A. (2006). Clasificaciones del aprendizaje híbrido y criterios de buenas prácticas universitarias. Costa Rica: Universidad Nacional de Costa Rica.

Stahl, G., Koschmann, T., \& Suthers, D. (2006). Computer-supported collaborative learning: An historical perspective. En R. K. Sawyer (Ed.), Cambridge handbook of the learning sciences (pp. 409-426). Cambridge: Cambridge University Press.

Tedesco, J. C. (2008) Las tic en la agenda de la política educativa. En J. C. Tedesco, N. C. Burbules, J. J. Brunner, E. Martín, P. Hepp, J. Morrissey, E. Duro, C. Magadán... I. Aguerrondo (Coords.), Las TiC. Del aula a la agenda política (pp. 31-40). Buenos Aires: Fondo de las Naciones Unidas para la Infancia. 
Guadalupe Álvarez, Lorena Bassa, Alejo González López Ledesma

Torres, P., \& Rama, C. (2010). La educación superior a distancia en América Latina y el Caribe. Santa Catarina: Unisul.

Vasilachis, I. (Coord.). (2006). Estrategias de una investigación cualitativa. Buenos Aires: Gedisa. 